\title{
Convergence and Aperiodicity in Fuzzy Cellular Automata: Revisiting Rule 90
}

\author{
P. Flocchini* $\quad$ F. Geurts ${ }^{\dagger} \quad$ A. Mingarelli M. Santoro $^{\S}$
}

\begin{abstract}
In this paper we consider a continuous version of cellular automata (Fuzzy CA) obtained by "fuzzification" of the disjunctive normal form which describes the corresponding Boolean rule. We concentrate on Fuzzy rule 90, whose Boolean version has deserved some attention for the complex patterns it generates. We show that the behavior of Fuzzy rule 90 is very simple, in that the system always converges to a fixed point. In the case of finite support configurations, we also show aperiodicity of every temporal sequences, extending and complementing Jen's result on aperiodicity of Boolean rule 90. We finally show and analyze the remarkable fact that, depending on the level of state-discreteness used to visualize the dynamics of Fuzzy 90, the display might show (after a transient) the well known complex Boolean behavior instead of the (correct) convergence to a fixed point. The results of the analysis lead not only to a caveat on the dangers of visualization, but also an unexpected explanation of the dynamics of Boolean rule 90 .
\end{abstract}

\section{Introduction}

The existing models for spatially extended systems have been extensively studied and applications have been found in a variety of contexts. For example, Boolean cellular automata (CA) have been introduced by Von Neumann as models of selforganizing/reproducing behaviors [15] and their applications range from ecology to theoretical computer science (e.g., [5, 7, 13, 16]); Coupled Map Lattices (CML) have been introduced by Kaneko as simple models with the features of spatiotemporal chaos, and have now applications in many different areas like fluid dynamics, biology, chemistry, etc. (e.g., $[1,11,12])$.

\footnotetext{
${ }^{*}$ University of Ottawa, School of Information Technology and Engineering, 150 Louis Pasteur, Ottawa, Canada. flocchin@site.uottawa.ca

†Département d'Informatique, Université Libre de Bruxelles, CP212, Boulevard du Triomphe, 1050 Bruxelles, Belgium, fgeurts@ulb.ac.be.

¥Department of Mathematics and Statistics, Carleton University, 1125 Colonel By Drive, 538 Herzberg Building, Ottawa K1S 5B6, Canada, amingare@math.carleton.ca.

${ }^{\S}$ School of Computer Science, Carleton University, 1125 Colonel By Drive, 538 Herzberg Building, Ottawa K1S 5B6, Canada, santoro@scs.carleton.ca.
} 
These models correspond to different levels of "discreteness"; for example, Partial Differential Equations represent the continuous extreme (being continuous in space, time and states), Cellular Automata represent the discrete extreme (being discrete in space, time, and state), Coupled Map Lattices are somehow in between (being discrete in space, time, but continuous in state).

An interesting area of investigation is the relationship among the different levels of discretization; in particular, some studies have been done to approximate CML by CA; i.e., to "discretize" some types of CML (e.g., $[2,3])$.

Fuzzy $C A s$ are an attempt to perform the reverse process; i.e., to start from a CA and "fuzzify" the disjunctive normal form which describes its rule [4]. Introduced to study the impact that state-discretization has on the behavior of these systems, they have been used to investigate the result of perturbations (e.g. noisy sources, computation errors, mutations, etc.) on the evolution of Boolean CA [6]. In this sense, this continuous-state CA model is a particular case of CML.

The goal of our work is the analytical study of dynamical properties of Fuzzy CA rules which generalize Boolean CAs. In this paper, we focus on Rule 90; its Boolean evolution, although linear, is considered to be complex and has been object of extensive investigation (e.g., see $[9,10,14]$ ).

We first show that the behavior of the Fuzzy version, which has never been studied before, is actually very simple: independently of initial configurations, the system converges to a fixed homogeneous configuration. In the particular case of finite support configurations, we generalize Jen's result [10, Prop. 2] on aperiodic sequences in Boolean Rule 90: we prove aperiodicity of temporal sequences in each site of the spatio-temporal diagram of Fuzzy Rule 90, we actually show periodicity also in every diagonal and even in every nontrivial temporal sequence.

We then observe an interesting behavior of the rule in the visualization process. When visualizing the space-time diagram of Fuzzy rule 90, the interval $[0,1]$ must be discretized, since only a finite number of states can obviously be represented. We show that this discretization process, inevitable for displaying the behavior of the rule, is however very dangerous in that it could mislead the observer by showing a totally incorrect dynamics. In fact, when the fixed point $\frac{1}{2}$ is an extreme of discretization interval, the space-time diagram of Fuzzy rule 90 shows fractal-like patterns, although the system actually converges to the fixed point.

We show that, such a wrong display, due to a certain discretization of the state space, after a short transient, surprisingly shows the exact same behavior of Boolean rule 90. Our analysis of this phenomenon provides an explanation about the dynamics of Boolean rule 90: its complex patterns describe the oscillations of Fuzzy Rule 90 around its fixed point. 


\section{Basic definitions}

A cellular automaton is a collection of cells arranged on a graph. All cells share the same local space (i.e., the set of values cells range in), the same neighborhood structure (i.e., the cells to which a cell is connected), and the same local function (i.e., the function defining the effect of neighbors on each cell, also called transition function or rule). The global evolution is defined by the synchronous update of all values according to the local function applied to the neighborhood of each cell. A configuration of the automaton is a description of all cell values.

Given a linear bi-infinite lattice of cells, the local Boolean space $\{0,1\}$, the neighborhood structure $\langle$ left neighbor, itself, right neighbor $\rangle$, and a local rule $g:\{0,1\}^{3} \mapsto\{0,1\}$, the global dynamics of an elementary $C A$ is defined by:

$$
\begin{array}{ll}
f: & \{0,1\}^{\mathbb{Z}} \mapsto\{0,1\}^{\mathbb{Z}} \\
\text { s.t. } & \forall i \in \mathbb{Z}, f(x)_{i}=g\left(x_{i-1}, x_{i}, x_{i+1}\right) .
\end{array}
$$

The local rule is defined by the 8 possible local configurations a cell can detect in its direct neighborhood:

$$
(000,001,010,011,100,101,110,111) \rightarrow\left(r_{0}, \cdots, r_{7}\right),
$$

where each triplet represents a local configuration of the left neighbor, the cell itself, and the right neighbor. In general, the value $\sum_{i=0: 7} 2^{i} r_{i}$ is used as the name of the rule. The local rule of any Boolean CA is canonically expressed as a disjunctive normal form:

$$
g\left(x_{1}, x_{2}, x_{3}\right)=\vee_{i \mid r_{i}=1} \wedge_{j=1: 3} x_{j}^{d_{i j}}
$$

where $d_{i j}$ is the $j$-th digit, from left to right, of the binary expression of $i$, and $x^{0}$ (resp. $x^{1}$ ) stands for $\neg x$ (resp. $x$ ).

Definition 1 A Fuzzy CA is obtained by fuzzification of the local function of a Boolean CA: in the disjunctive normal form, $(a \vee b)$ is replaced by $(a+b),(a \wedge b)$ by $(a b)$, and $(\neg a)$ by $(1-a)$. The resulting local rule is a real-valued function simulating the original function on $\{0,1\}^{3}$, with $l(a, 0)=1-a$ and $l(a, 1)=a$ :

$$
\begin{array}{ll}
g: \quad & {[0,1]^{3} \mapsto[0,1]} \\
\text { s.t. } & g\left(x_{1}, x_{2}, x_{3}\right)=\sum_{i=0: 7} r_{i} \prod_{j=1: 3} l\left(x_{j}, d_{i, j}\right) .
\end{array}
$$

The usual fuzzification of the expression $a \vee b$ is $\max \{1, a+b\}$ so as to ensure that the result is not larger than 1 . Note, however, that taking $(a+b)$ for the CA fuzzification does not lead to values greater than 1 since the sum of all the expressions for Rule 255 is 1 , and so every possible partial sum must be bounded by 1 .

Example 2 Consider rule $14=2+4+8$ :

$$
(000,001,010,011,100,101,110,111) \rightarrow(0,1,1,1,0,0,0,0) .
$$


The canonical expression of rule 14 is:

$$
g_{14}\left(x_{1}, x_{2}, x_{3}\right)=\left(\neg x_{1} \wedge \neg x_{2} \wedge x_{3}\right) \vee\left(\neg x_{1} \wedge x_{2} \wedge \neg x_{3}\right) \vee\left(\neg x_{1} \wedge x_{2} \wedge x_{3}\right) .
$$

The fuzzification process after simplification yields:

$$
g_{14}\left(x_{1}, x_{2}, x_{3}\right)=\left(1-x_{1}\right) \cdot\left(x_{2}+x_{3}-x_{2} \cdot x_{3}\right) .
$$

In the rest of this paper, unless specified otherwise, we will study Fuzzy Rule 90 , defined by the local function

$$
\begin{array}{ll}
g: & {[0,1]^{3} \mapsto[0,1]} \\
\text { s.t. } & g(x, y, z)=x+z-2 x z .
\end{array}
$$

\section{Homogeneous configurations}

The first step of our analysis consists in a strong assumption on the values undertaken by the fuzzy cells of the automaton we consider: they are all initialized to the same value and, as the global dynamics is homogeneous, all subsequent configurations are homogeneous, too. This reduces the infinite-dimensional system to a a one-dimensional one, the dynamics of which is analyzed in a straightforward way. The motivation is to get a clear idea of the local process.

The reduced function is

$$
\begin{array}{ll}
h: & {[0,1] \mapsto[0,1]} \\
\text { s.t. } & h(x)=2 x(1-x) .
\end{array}
$$

It has two fixed points: 0 and $\frac{1}{2}$. The absolute value of the first derivative $h^{\prime}(x)=2(1-2 x)$ evaluated in each of these fixed points gives $\left|h^{\prime}(0)\right|=2$ and $\left|h^{\prime}\left(\frac{1}{2}\right)\right|=0$. Thus, 0 turns out to be repelling, and $\frac{1}{2}$ is an attractor. The second order Taylor expansion of $h$ around $x$ is

$$
\begin{aligned}
h(x+u) & =h(x)+h^{\prime}(x) u+\frac{1}{2} h^{\prime \prime}(x) u^{2} \\
& =2 x(1-x)+(2-4 x) u-2 u^{2}
\end{aligned}
$$

and, around the fixed points, we have $h(u) \approx 2 u$ and $h\left(\frac{1}{2}+u\right)=\frac{1}{2}-2 u^{2}$. Initial conditions close to zero are essentially multiplied by two, and attracted by $\frac{1}{2}$ : for any $u \in\left(-\frac{1}{2}, \frac{1}{2}\right), h\left(\frac{1}{2}+u\right)$ is strictly closer to $\frac{1}{2}$ than $\frac{1}{2}+u$.

Thus, this one-dimensional system behaves in a very simple way: 0 is a repelling fixed point; 1 is attracted to 0 in one iteration; $\frac{1}{2}$ is an attracting fixed point whose basin is the open interval $(0,1)$.

\section{Single values in zero backgrounds}

In this second analysis, all cells but one are initially set to 0 . This step is motivated by the classical analysis of finite support configurations of Boolean 


\begin{tabular}{c|lcccccccc} 
& \multicolumn{10}{|c}{ Local states } \\
Time & $\cdots$ & -3 & -2 & -1 & 0 & 1 & 2 & 3 & $\cdots$ \\
\hline 0 & $\cdots$ & 0 & 0 & 0 & $\frac{1}{4}$ & 0 & 0 & 0 & $\cdots$ \\
1 & $\cdots$ & 0 & 0 & $\frac{1}{4}$ & 0 & $\frac{1}{4}$ & 0 & 0 & $\cdots$ \\
2 & $\cdots$ & 0 & $\frac{1}{4}$ & 0 & $\frac{3}{8}$ & 0 & $\frac{1}{4}$ & 0 & $\cdots$ \\
3 & $\cdots$ & $\frac{1}{4}$ & 0 & $\frac{7}{16}$ & 0 & $\frac{7}{16}$ & 0 & $\frac{1}{4}$ & $\cdots$ \\
4 & $\cdots$ & 0 & $\frac{15}{32}$ & 0 & $\frac{63}{128}$ & 0 & $\frac{15}{32}$ & 0 & $\cdots$ \\
$\vdots$ & & & & & $\vdots$ & & & &
\end{tabular}

Table 1: Evolution from $\frac{1}{4}$ in a zero background.

CA [10]. For example, starting from a single value $a=\frac{1}{4}$ in a zero background, the spatio-temporal evolution is represented in Table 1.

More abstractly, assuming $a \neq 0$ and $g_{a}(x)=g(x, \bullet, a)=g(a, \bullet, x)=a+$ $x(1-2 a)$, we have the evolution of Table 2 . What is the rule underlying these

\begin{tabular}{c|ccccccccc} 
& \multicolumn{7}{|c}{ Local states } \\
Time & $\cdots$ & -3 & -2 & -1 & 0 & 1 & 2 & 3 & $\cdots$ \\
\hline 0 & $\cdots$ & 0 & 0 & 0 & $a$ & 0 & 0 & 0 & $\cdots$ \\
1 & $\cdots$ & 0 & 0 & $a$ & 0 & $a$ & 0 & 0 & $\cdots$ \\
2 & $\cdots$ & 0 & $a$ & 0 & $h(a)$ & 0 & $a$ & 0 & $\cdots$ \\
3 & $\cdots$ & $a$ & 0 & $g_{a}(h(a))$ & 0 & $g_{a}(h(a))$ & 0 & $a$ & $\cdots$ \\
4 & $\cdots$ & 0 & $g_{a}^{2}(h(a))$ & 0 & $h\left(g_{a}(h(a))\right)$ & 0 & $g_{a}^{2}(h(a))$ & 0 & $\cdots$ \\
$\vdots$ & & & & & $\vdots$ & & & &
\end{tabular}

Table 2: Evolution from $a$ in a zero background.

numbers, if any?

Definition 3 The spatio-temporal diagram from an initial configuration $x^{0}$ is the double sequence $\left(x_{i}^{t}\right)_{i \in \mathbb{Z}, t \in \mathbb{N}}$ where $t$ expresses time steps, and $i$ denotes cell indices.

Definition 4 The $j$ th diagonal is the sequence $\left(x_{i}^{i+2(j-1)}\right)_{i \geq 0}$.

Definition 5 The light cone from a cell $x_{i}^{t}$ is the set $\left\{x_{j}^{t+p} \mid p \geq 0 \wedge j \in\right.$ $\{i-p, \cdots, i+p\}\}$.

Proposition 6 The second diagonal of the spatio-temporal diagram obtained by the evolution of Fuzzy Rule 90 from a single value $a \in(0,1)$ in a zero background converges to $\frac{1}{2}$. 
Proof. The first diagonal starting from the central non-zero value $a$ is uniformly equal to $a$. The second diagonal, from $h(a)$, can be obtained by successive iterations of $g_{a}(x)$. It has exactly one fixed point $x=\frac{1}{2}$, independently of $a$. The absolute value of the slope of this linear function is smaller than one iff $a \in(0,1)$. This means that the second diagonal converges to $\frac{1}{2}$ as time goes to infinity.

Let $f(t, i)=\left(\begin{array}{c}t \\ \frac{t+i}{2}\end{array}\right)$, where $\left(\begin{array}{c}a \\ b\end{array}\right)=\frac{a !}{b !(a-b) !}$.

Proposition 7 The spatio-temporal diagram from a single value $a \in(0,1)$ in a zero background is explicitly given by: $\forall t \in \mathbb{N}$,

$$
x_{i}^{t}= \begin{cases}\frac{1}{2}\left(1-(1-2 a)^{f(t, i)}\right) & \text { if } t+i \text { is even and } i \in\{-t, \cdots, t\} \\ 0 & \text { otherwise }\end{cases}
$$

Proof. By induction. The first case is straightforward: $x_{0}^{0}=a$ and $\forall i \neq$ $0, x_{i}^{0}=0$. The inductive case is twofold.

- If $(t+1)+i$ is odd, $i>t+1$ or $i<-t-1$, then $x_{i-1}^{t}=x_{i+1}^{t}=0$, whence $x_{i}^{t+1}=0$.

- Otherwise, $(t+1)+i$ and $t+(i-1)$ are even, and $i \in\{-t-1, \cdots, t+1\}$. Thus, if $-(t-1) \leq i \leq t-1$, then

$$
\begin{aligned}
x_{i}^{t+1} & =g\left(x_{i-1}^{t}, \bullet, x_{i+1}^{t}\right) \\
& =\frac{1}{2}\left(1-(1-2 a)^{f(t, i-1)+f(t, i+1)}\right) \\
& =\frac{1}{2}\left(1-(1-2 a)^{f(t+1, i)}\right) .
\end{aligned}
$$

The two limit cases are $i-1=-t-2$ and, symmetrically, $i+1=t+2$. Let us examine the first one:

$$
\begin{aligned}
x_{i}^{t+1} & =x_{-t-1}^{t+1}=g\left(x_{-t-2}^{t}, \bullet, x_{-t}^{t}\right)=g\left(0, \bullet, x_{-t}^{t}\right)=x_{-t}^{t} \\
& =\frac{1}{2}\left(1-(1-2 a)^{f(t,-t)}\right)=a .
\end{aligned}
$$

Thus, any infinite sequence of non-zero terms in the diagram converges to $\frac{1}{2}$, provided that it is embedded in the light cone originating from the central $a$, and the sequence of non-zero time steps tends to infinity. The central column, any diagonal, any sequence containing horizontal segments or even backward loops, they all converge to $\frac{1}{2}$.

Corollary 8 Let $x_{0}^{0}=a \in(0,1), x_{i \neq 0}^{0}=0, i: \mathbb{N} \mapsto \mathbb{Z}$ and $\tau: \mathbb{N} \mapsto \mathbb{N}$ be two functions such that $i(0)=\tau(0)=0,|i(j)| \leq \tau(j), x_{i(j)}^{\tau(j)} \neq 0$, and $\lim _{j \rightarrow \infty} \tau(j)=\infty$. Then, the sequence $\left(x_{i(j)}^{\tau(j)}\right)_{j \in \mathbb{N}}$ converges to $\frac{1}{2}$. 
Remark 9 Exactly as in nontrivial evolutions of Boolean Rule 90 [10], any nontrivial infinite sequence of states taken in the spatio-temporal evolution of Fuzzy Rule 90 from a single value in a zero background is aperiodic, due to Proposition 7. On the other hand, any such sequence converges to a specific value, here $\frac{1}{2}$, which was not the case of Boolean Rule 90 .

Remark 10 If the initial configuration contains two consecutive values, $a$ and $b$, in a zero background, the result is obtained by superposition of the individual diagrams obtained from $a$ and $b$. Let $\left(x_{i}^{t}\right)$ be such that $x_{0}^{0}=a$ and $\forall i \neq 0, x_{i}^{0}=0$, $\left(y_{i}^{t}\right)$ be such that $y_{1}^{0}=b$ and $\forall i \neq 1, y_{i}^{0}=0$, then $\left(z_{i}^{t}=x_{i}^{t}+y_{i}^{t}\right)$ is the diagram starting from $z_{0}^{0}=a, z_{1}^{0}=b$ and $\forall i \in \mathbb{Z} \backslash\{0,1\}, z_{i}^{0}=0$. Unfortunately, this property cannot be extended to larger finite support configurations.

\section{$5 \quad$ Infinite heterogeneous configurations}

Here, we extend the analysis to evolutions starting from heterogeneous configurations, i.e., initial values chosen arbitrarily in $(0,1)$. With such a weak assumption, we are, of course, not able to derive the closed-form expression of all $x_{i}^{t}$, but the last result mentioned in $\S 4$ still holds: we prove that any sequence of non-zero terms in the spatio-temporal diagram converges to $\frac{1}{2}$. We first establish useful lemmas, we then prove the main result.

Let us rewrite the local function $g$ as follows:

$$
\phi(x, y)=g\left(\frac{1}{2}+x, \bullet, \frac{1}{2}+y\right)=\frac{1}{2}-2 x y .
$$

Lemma 11 The function $g$ is contracting around $\frac{1}{2}$ :

$$
\forall x, y \in\left(0, \frac{1}{2}\right) \cup\left(\frac{1}{2}, 1\right),\left|g(x, \bullet, y)-\frac{1}{2}\right|<\min \left\{\left|x-\frac{1}{2}\right|,\left|y-\frac{1}{2}\right|\right\} .
$$

Proof. Let us change the variables: $x=\frac{1}{2}+v, y=\frac{1}{2}+w$, and rewrite the lefthand side expression: $\left|g(x, \bullet, y)-\frac{1}{2}\right|=\left|g\left(\frac{1}{2}+v, \bullet, \frac{1}{2}+w\right)-\frac{1}{2}\right|=\left|\phi(v, w)-\frac{1}{2}\right|=$ $|2 v w|$. Since $0<|w|<\frac{1}{2}$, we have $|2 v w|<|v|=\left|x-\frac{1}{2}\right|$. Symmetrically, $|2 v w|<|w|=\left|y-\frac{1}{2}\right|$.

Lemma 12 The function $g$ is $k$-contracting around $\frac{1}{2}$ on $\left[\frac{1-k}{2}, \frac{1+k}{2}\right]$ :

$$
\forall x, y \in\left[\frac{1-k}{2}, \frac{1+k}{2}\right],\left|g(x, \bullet, y)-\frac{1}{2}\right| \leq k \min \left\{\left|x-\frac{1}{2}\right|,\left|y-\frac{1}{2}\right|\right\} .
$$

Proof. Changing variables as in the previous lemma, we have $\left|g(x, \bullet, y)-\frac{1}{2}\right|=$ $|2 v w| \leq 2 \frac{k}{2}|v|$, since $y=w+\frac{1}{2} \in\left[\frac{1-k}{2}, \frac{1+k}{2}\right]$.

The next example gives a convergence rate for purely temporal sequences of the diagram (i.e., columns), where every other row is ignored (since $g$ does not depend on its central argument). The proof easily follows from the previous lemma. 
Example 13 If $x^{0} \in(0,1)^{\mathbb{Z}}$ and $x_{0}^{0} \in\left[\frac{1-k}{2}, \frac{1+k}{2}\right]$, then $\left|x_{0}^{2}-\frac{1}{2}\right| \leq k^{2}\left|x_{0}^{0}-\frac{1}{2}\right|$, and the sequence $\left(x_{0}^{2 t}\right)_{t \in \mathbb{N}}$ converges to $\frac{1}{2}$.

If connected paths are considered in the spatio-temporal diagram, the convergence rate is $k$.

Example 14 If $x^{0} \in(0,1)^{\mathbb{Z}}$ and $x_{0}^{0} \in\left[\frac{1-k}{2}, \frac{1+k}{2}\right]$, then $\left|x_{ \pm 1}^{1}-\frac{1}{2}\right| \leq k\left|x_{0}^{0}-\frac{1}{2}\right|$, and any connected path $\left(x_{i(j)}^{j}\right)_{j \in \mathbb{N}}$ such that $i(0)=0$ and $\forall j \in \mathbb{N}, i(j+1)=i(j) \pm 1$, converges to $\frac{1}{2}$.

Finally, using Lemma 12 again, we generalize Corollary 8: any path in the spatio-temporal can be considered, and still lead to the same conclusion.

Theorem 15 Let $x^{0} \in[0,1]^{\mathbb{Z}}$ be such that $\liminf x_{i}^{0}>0$ and $\lim \sup x_{i}^{0}<1$, $i: \mathbb{N} \mapsto \mathbb{Z}$ and $\tau: \mathbb{N} \mapsto \mathbb{N}$ be two functions, $\tau(0)=0$, and $\lim _{j \rightarrow \infty} \tau(j)=\infty$. Then, the sequence $\left(x_{i(j)}^{\tau(j)}\right)_{j \in \mathbb{N}}$ converges to $\frac{1}{2}$.

Proof. Let us define $k=2 \max \left\{\left|\lim \inf x_{i}^{0}-\frac{1}{2}\right|,\left|\lim \sup x_{i}^{0}-\frac{1}{2}\right|\right\}$. We have of course $0<k<1$ and, $\forall i, x_{i}^{0} \in\left[\frac{1-k}{2}, \frac{1+k}{2}\right]$. Thus, $\left|x_{i \pm 1}^{1}-\frac{1}{2}\right| \leq k\left|x_{i}^{0}-\frac{1}{2}\right| \leq k \frac{k}{2}$. The evolution does not depend on position $i$ anymore; in fact, $\forall t, i,\left|x_{i}^{t}-\frac{1}{2}\right| \leq$ $\frac{1}{2} k^{t+1}$, whence the result.

Indeed, the function $g$ may be rewritten in the form $1-2 g(x, z)=(1-$ $2 x)(1-2 z)$, and this suggests that a different proof of the convergence to $\frac{1}{2}$ may be obtained by a simple variable change. Since we know that $1-2 g(x, z)=(1-$ $2 x)(1-2 z)$, applying this rule $(n-1)$ times to a finite string $a_{1} a_{2} a_{3} \ldots a_{n}$, we have:

$$
a_{1} a_{2}\left(\begin{array}{c}
n-1 \\
1
\end{array}\right) a_{3}\left(\begin{array}{c}
n-1 \\
2
\end{array}\right) \ldots a_{n-1}\left(\begin{array}{c}
n-1 \\
n-2
\end{array}\right) a_{n} .
$$

If all the $a_{i}$ 's are bounded by $1-\varepsilon$, this is bounded by $(1-\varepsilon)^{2^{n-1}}$. This clearly goes to 0 exponentially fast, implying convergence to $\frac{1}{2}$ in the original variables.

\section{On the observation precision and the Boolean case}

In the previous sections we have shown that that Fuzzy Rule 90 has a very simple behavior: it attracts everything to $\frac{1}{2}$. The result leads to the intriguing question: where do the complex patterns in its Boolean evolution come from?

We know that Fuzzy Rule 90 attracts everything to $\frac{1}{2}$. We also know that the values alternate around this point as they get closer to it: this is due to the minus sign appearing in equation (1). The corresponding "rule table" is detailed in Table 3 left. 

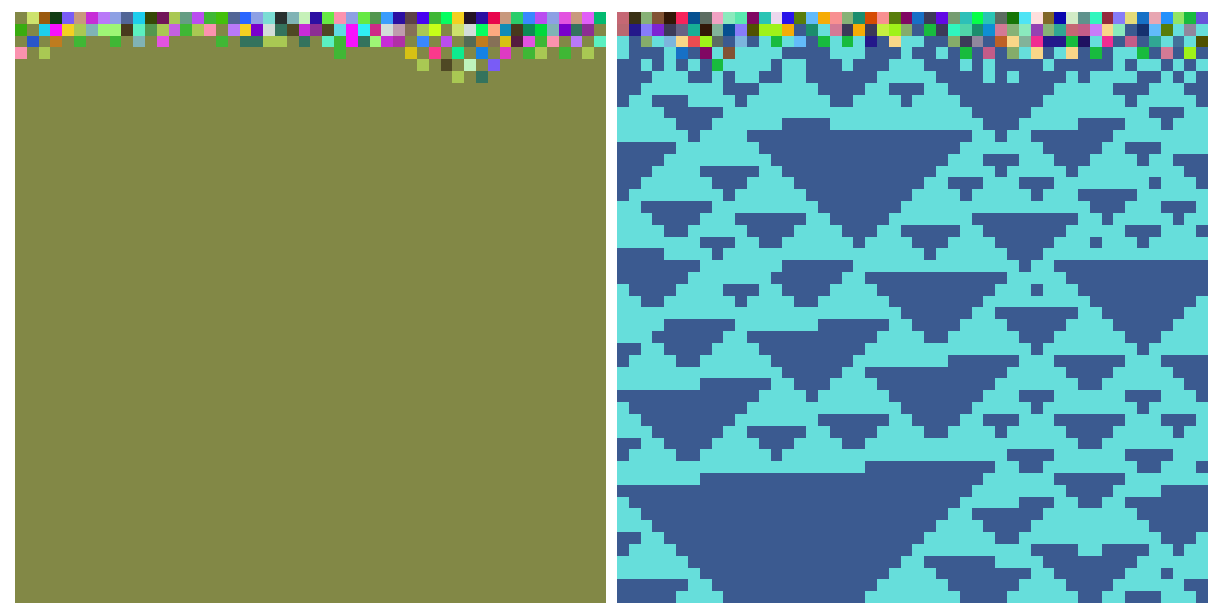

Figure 1: Evolution of Fuzzy rule 90. Grey levels indicate different value ranges. Times evolves from top to bottom. Left: 127 discretization intervals have been used and the fixed point $\frac{1}{2}$ is in the middle of the central interval. Right: 128 discretization intervals have been used and the fixed point $\frac{1}{2}$ is on the border of one of the central intervals.

Consider a partition of the interval $[0,1]$ in a finite number of subintervals, used as coarse-grained approximations of the real numbers. Such a discretization is necessary for visualizing the space-time diagram of Fuzzy rule 90. The graphical representation will strongly depends on whether $\frac{1}{2}$ is in the middle or on a border of one of the discretization subintervals.

If the fixed point $\frac{1}{2}$ is in the middle of a subinterval, the observation becomes homogenenous after a few steps, as all the iterations get quickly very close to the fixed point (see Fig. 1 left). On the contrary, if the fixed point is on the border of some subinterval, the values will alternate around the two subintervals bordering on the fixed point according to the rule of Table 3 left. Thus, the visualization will show the alternance between intervals (i.e., using different grey levels, depending upon whether they are smaller or greater than $\frac{1}{2}$ ) creating the seemingly "chaotic" triangular patterns found in the Boolean evolution (see Fig. 1 right); this is exactly what happens in the binary case, when the interval $[0,1]$ is divided into two subintervals. In other words, if the fixed point is on the border of some subinterval, the visualization displays a consequence of the discretization process and misleads the observer not showing the inherent convergence to $\frac{1}{2}$ which is the basic behavior of Fuzzy Rule 90.

This leads to an observation on the observation precision. In fact, as we have seen, the same dynamics can be observed as very simple or totally complex, depending upon whether the attracting fixed point falls in the interior or on the border of some discretization interval. In other words, the observed behaviour is an artifact of the visualization toos used. This very important phenomenon is not a specific feature of Fuzzy Rule 90; actually, when looking 


\begin{tabular}{|c|c|c|c|c|c|c|c|}
\hline \multicolumn{4}{|c|}{ Behavior around $\frac{1}{2}$} & \multicolumn{4}{|c|}{ Boolean Rule 90} \\
\hline$x$ & $y$ & $z$ & $g(x, y, z)$ & $x$ & $y$ & $z$ & $g(x, y, z)$ \\
\hline- & - & - & $\overline{-}$ & $\overline{0}$ & 0 & 0 & 0 \\
\hline- & - & + & + & 0 & 0 & 1 & 1 \\
\hline- & + & - & - & 0 & 1 & 0 & 0 \\
\hline- & + & + & + & 0 & 1 & 1 & 1 \\
\hline+ & - & - & + & 1 & 0 & 0 & 1 \\
\hline+ & - & + & - & 1 & 0 & 1 & 0 \\
\hline+ & + & - & + & 1 & 1 & 0 & 1 \\
\hline+ & + & + & - & 1 & 1 & 1 & 0 \\
\hline
\end{tabular}

Table 3: Left: Behavior around $\frac{1}{2}$. The rule table is obtained from equation (1): - (resp. +) stands for "smaller (resp. greater) than $\frac{1}{2}$ ". Right: Rule table of Boolean Rule 90

at the behavior of any continous system by simulation, the descriptive precision used is of utmost importance on the observed complexity and it deserves a deep investigation. This indicates the limits and the dangers of visualization tools and of experimental analysis.

Another very important observation concerns the question we posed at the beginning of this section: where the "complex" patterns of linear Boolean rule 90 come from? In the Fuzzy case, we have seen that, if $\frac{1}{2}$ is the extreme of one of the discretization subintervals, the display hows, after a short transient, the exact same behavior of Boolean rule 90 (see Fig. 2). This surprising and remarkable observation is actually explained by the fact that the table of Boolean Rule 90 (see Table 3 rigth) is exactly the same as the one describing the alternance around the fixed-point of Fuzzy Rule 90 (see Table 3 left) where - (resp. +) is replaced by 0 (resp. 1). This provides the first explanation of the "complex" nature of the evolution of the Boolean rule: The well-known observed dynamics of Boolean Rule 90 (see Fig. 2) describes the behavior of Fuzzy Rule 90 around its fixed point.

\section{Concluding Remarks}

An interesting open direction is to find general techniques for analyzing the Fuzzy rules' behaviors and to apply them to classes of rules instead of having to perform case-by-case analysis.

Some transformations and operations on the rules (which preserve qualitative and/or quantitative dynamic properties) could be used to extend the class of rules to which our method applies. Infact, building homomorphisms between known and new systems, composing known rules to obtain new ones, and combining individual properties to get homomorphically global ones, the methods described in this paper can be applied, with minor modifications, to the Fuzzy Rules $60,102,153,165$, and 195 where we find the same convergence properties. 


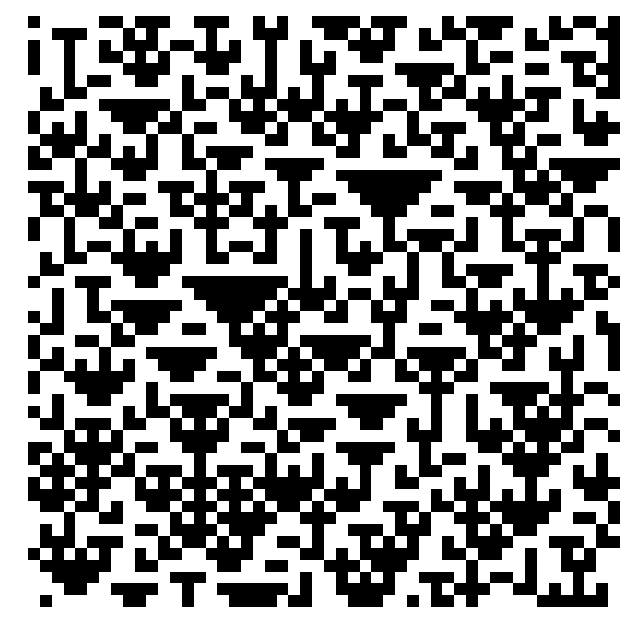

Figure 2: Evolution of Boolean rule 90 from a random initial configuration. The black pixels correspond to the value 0 , the white pixels correspond to 1 .

Acknowledgements. P.F. is supported in part by NSERC and FCAR Canada; F.G. acknowledges the financial support of FNRS and CFB Belgium when visiting Carleton University; A.M. and N.S. are supported in part by NSERC.

\section{References}

[1] L.A. Bunimovich Coupled Map Lattices: one Step Forward and two Steps Back. Physica D, 86:248-255, 1995.

[2] H. Chaté, P. Manneville Coupled map lattices as cellular automata. J. Stat. Phys., 56:357-370, 1989.

[3] H. Chaté, P. Manneville Criticality in cellular automata. Physica D, 45:122-135, 1990.

[4] G. Cattaneo, P. Flocchini, G. Mauri, and N. Santoro. Cellular automata in fuzzy backgrounds. Physica D, 105:105-120, 1997.

[5] K. Culik II and S. Yu. Undecidability of CA classification schemes. Complex Systems, 2:177-190, 1988.

[6] P. Flocchini and N. Santoro. The chaotic evolution of information in the interaction between knowledge and uncertainty. In Complex Systems: Mechanism of Adaptation, 337-343. IOS Press, 1994.

[7] M. Garzon. Models of Massive Parallelism. Analysis of Cellular Automata and Neural Networks. Springer-Verlag, 1995. 
[8] F. Geurts. Abstract Compositional Analysis of Iterated Relations. SpringerVerlag, 1998.

[9] J. E. Hanson and J. P. Crutchfield. The attractor-basin portrait of a cellular automaton. Technical Report 91-02-012, Santa Fe Institute, 1991.

[10] E. Jen. Aperiodicity in one-dimensional cellular automata. Physica D, 45:3-18, 1990.

[11] K. Kaneko (Ed.), Theory and Application of Coupled Map Lattices. John Wiley \& Sons Ltd, 1993.

[12] G. Keller, M. Künzle, T. Nowiki Some Phase Transitions in Coupled Map Lattices. Physica D 59:39-51, 1992.

[13] C. G. Langton. Studying artificial life with cellular automata. In Evolution, Games, and Learning. North Holland, 1986.

[14] H. Y. Lee and Y. Kawahara. On dynamical behaviors of cellular automata CA-60. Bulletin of Informatics and Cybernetics, 25(1-2):21-25, 1992.

[15] J. Von Neumann. Theory of Self-Reproducing Automata. University of Illinois Press, Urbana, 1966.

[16] S. Wolfram. Cellular Automata and Complexity. Collected Papers. World Scientific, 1994. 